\title{
Disseminating a Large Amount of Data to Vehicular Network in an Urban Area
}

\author{
Mina Taheri and Faramarz Hendesi \\ Department of Electrical and Computer Engineering, Isfahan University of Technology, Esfahan 84156-83111, Iran \\ Correspondence should be addressed to Mina Taheri, mina.taheri.h@gmail.com
}

Received 13 August 2010; Accepted 31 December 2010

Academic Editor: Shaharuddin Salleh

Copyright ( $) 2011 \mathrm{M}$. Taheri and F. Hendesi. This is an open access article distributed under the Creative Commons Attribution License, which permits unrestricted use, distribution, and reproduction in any medium, provided the original work is properly cited.

The problem of distributing a large amount of data from multiple sources in an urban area is investigated. We explore an opportunistic approach for information collection, in which a vehicle obtains information about resources from encountered vehicles. This protocol could be applied in both dense and sparse vehicular networks. Due to the highly dynamic nature of the underlying vehicular network topology, we depart from architectures requiring centralized coordination, reliable MAC scheduling, or global network state knowledge, and instead adopt a distributed paradigm with simple protocols. In other words, a reliable dissemination is introduced from multiple sources when each node in the network shares a limited amount of its resources for cooperating with others. By using rateless coding at the Road Side Unit (RSU) and using vehicles as data carriers, an efficient way to achieve reliable dissemination to all nodes (even disconnected clusters in the network) is described.

\section{Introduction}

Recently, many researches have been done to use vehicular ad hoc networks (VANET) for safety and commercial purposes $[1-3]$.

The integration of communication technology in stateof-the-art vehicles has begun years ago: car phones and internet access based on cellular technologies as well as Bluetooth adapters for the integration of mobile devices are popular examples. However, the direct communication between vehicles using an ad hoc network, referred to as intervehicle communication (IVC) or vehicle ad hoc networks (VANETs), is a relatively new approach. Compared to a cellular system, IVC has three key advantages: lower latency due to direct communication, broader coverage, and having no service fee.

Recently, the promises of wireless communications to support vehicular safety applications have led to several research projects around world: the Vehicle Safety Communications Consortium, developing the DSRC Technology (USA), the Internet ITS Consortium (Japan), the PREVENT project (Europe), and the "Network on Wheels" project (Germany) are some samples.
To cater to the emerging wireless communication needs with regard to vehicles, in July 2003, ASTM and IEEE adopted the Dedicated Short Range Communication (DSRC) standard (ASTM E 2213-03) [4]. The aim of this standard is to provide wireless communications capabilities for transportation applications within a $1000 \mathrm{~m}$ range at typical highway speeds. It provides seven $10 \mathrm{MHz}$ channels at the $5.9 \mathrm{GHz}$ licensed band for ITS applications, with different channels designated for different applications, including one specifically reserved for vehicle-to-vehicle communications. The specific properties of VANETs allow the development of attractive new services.

Vehicular communications have many different facets. Applications range from safety support [5] (e.g., collision warnings, slow-down warnings), to entertainment for passengers, to local news delivery and advertisement [6] (e.g., electronic toll collection, map download, video download, Internet transactions, Parking Space availability). The key parameter for providing these applications is message dissemination. According to above classification, the messages which are exchanged between vehicles can be categorized into three classes. 
(1) Event driven safety messages: which are the results of the detection of an unsafe situation, (e.g., a car crash, the proximity of vehicles at high speed, etc.).

(2) Periodic safety messages: also called beacon messages, are needed to make vehicles aware of their environment and also commercial purposes. Thus, they will be able to avoid emergency or unsafe situations even before they appear. Therefore, beacon messages essentially contain the state of the sending vehicle, that is, position, direction, speed, and so forth, and also aggregated data regarding the state of their neighbors.

(3) Comfort messages: all other types of data packets are included in comfort messages (e.g., data packets include internet access, video conferencing, etc.).

In this paper, a protocol which is able to disseminate a large amount of commercial data to urban areas from roadside units is proposed. This protocol can be used for both dense and sparse networks. Moreover, In previous works, neighbors are assumed to be recognized by beacons which are periodically sent by carriers. In this approach, there is no need to send these beacons.

The protocol should answer to these questions: when and how the act of forwarding should happen; in other words, how does the carrier decide to forward the message? Does it need to keep a copy message for itself?

The remainder of this paper is organized as follows. In Section 2, a brief review of previous works about distributing data in vehicular networks is propounded. In Section 3, we introduce the application of commercial advertisements in VANET. In Section 4, characteristics of vehicular ad hoc networks will be expressed. Section 5 reviews the DMRC method investigated in [7]. Our proposed scheme and simulation tools are described in Section 6. Finally, the paper is concluded in Section 7.

\section{Related Work}

Advertisements are one of the most important sources of revenue for companies. The advertisement application is a type of data dissemination from an information source to a large number of clients. In this work, we will take some steps to answer questions concerning data dissemination in the context of disseminating information packets from a large array of Road Side Units (RSU) to a bidirectional linear highway vehicular network. Vehicular ad hoc networks (VANETs) can be considered as a category of partitioned ad hoc networks [8]. Since density of vehicles is highly variable with space and time, the network changes from a sparsely disconnected to a densely connected in a short period of time. As a result of these topology variations, traditional routing and forwarding methods do not have a reasonable performance in VANET scenarios. In order to have message dissemination in partitioned ad hoc networks, the idea of Store-Carry-Forward (SCF) was proposed [1]. In SCF, a node carries information while there is not any other node in its vicinity. As soon as another node is detected, the forwarding phase starts. In recent years, several works have been done in using SCF which are more compatible with VANET environment. The most important of these works are SODAD [2], VADD [3], and MDDV [9]. A weak point in SCF is that sometimes the message transfer speed is limited by nodes' velocities. This increases end-to-end delay from a source to a destination. Fortunately, comfort applications of VANET are Delay Tolerant and applying SCF cannot be a major problem. Although SCF seems to be the proper answer for sparse scenarios, designing a routing protocol that can seamlessly handle the two extreme cases: well-connected networks and disconnected networks is still a challenge. In our proposed mechanism, an opportunistic approach of SCF is used. In opportunistic forwarding [10], message dissemination happens when a forwarding opportunity is detected. After forwarding a packet, a copy may be kept in the original node for further forwarding, if needed. For VANETs, opportunity can be defined as a situation where two vehicles meet each other. In [7], disseminating a large amount of data in highways is investigated. The characteristic of highways is that vehicles have approximately constant speed during their moving across the highways.

\section{Commercial Advertisement in Vanet}

Consider a downtown area with many stores and entertainment centers. Each store has different products and services. Some of products are on sale, some of the entertainment seats are available, and some foods are close to expiration date. Now consider many vehicles which carry many passengers in the downtown area. If store owners advertise their sale or activity information in nearby area, they have the opportunity to find some customers out of these passengers. With this motivation, any store is willing to use a special device to become a Roadside Advertising Unit (RSAU). In a conventional VANET, four primary data transfers can be assumed: (1) vehicle-to-vehicle (v2v) data transfer, (2) vehicle-to-roadside data transfer (v2r), (3) roadside-tovehicle date transfer $(\mathrm{r} 2 \mathrm{v})$, and (4) roadside-to-roadside date transfer $(\mathrm{r} 2 \mathrm{r})$.

In this work, we continued the DMRC [7] approach for urban area. Therefore, at first the DMRC scheme will be introduced and we develop it for the roads containing vehicles with various speeds and different traffic loads. In our approach, the typical traffic is advertisement data generated by roadside advertising units (RSAU). Each RSAU is equipped with a short range wireless broadcast point which broadcasts the advertisements to the vehicles $(\mathrm{r} 2 \mathrm{v})$. Vehicles are collecting these data when they are moving toward the RSAU and distributing the data when they are moving in opposite direction. In this paper, a new approach that merges the vehicle-to-vehicle and roadsideto-vehicle communication typologies in order to support reliable data dissemination in an urban area without the need of complex routing protocols is presented. Also our focus in this paper is on sparse networks. In the following, we first reminisce the characteristics of disconnected ad hoc networks. 


\section{Characteristics of Disconnected Vehicular Ad Hoc Networks}

Realizing that a Vehicular Ad hoc Network is prone to network fragmentation, it becomes essential to capture VANET's traffic characteristics for a better understanding of this phenomenon. Based on investigations in [11], it is observed that vehicles tend to move in clusters where two consecutive clusters of vehicles are normally separated by a relatively large distance. Also, it is observed that the probability distribution of the spacing between equipped vehicles in a network with can be approximated as an exponential distribution with parameter $\lambda_{s}$, given by

$$
f_{s}(s)=\lambda_{s} e^{-\lambda_{s} s} \text {. }
$$

4.1. Average Intracluster Spacing $\left(E\left[S_{\text {intra }}\right]\right)$. In this subsection, we are particularly interested in characterizing the intracluster spacing between adjacent vehicles $i$ and $i+1$ which travel in the same cluster. Since the two vehicles belong to the same cluster, the distance between them should be less than the transmission range $R$. Given that the intervehicle spacing $S$ has an exponential distribution, it follows that the Probability Distribution Function (PDF) of $S_{\text {intra }}$ can be expressed as

$$
f_{S_{\text {intra }}}\left(S_{\text {intra }}\right)=P_{r}[S \mid S \leq R]=\frac{\lambda_{s} e^{-\lambda_{S} S_{\text {intra }}}}{1-e^{-\lambda_{s} R}} .
$$

4.2. Average Inter-Cluster Spacing $\left(E\left[S_{\text {inter }}\right]\right)$. Obviously, in line with the concept of clusters, the distance between the last vehicle of the leading cluster and the first vehicle of the following cluster should be larger than transmission range $R$. Given that the interarrival spacing $S$ follows an exponential distribution, PDF of $S_{\text {intra }}$ can be expressed as

$$
f_{S_{\text {inter }}}\left(S_{\text {inter }}\right)=P_{r}[S \mid S>R]=\lambda_{s} e^{-\lambda_{s}\left(S_{\text {inter }}-R\right)} .
$$

Based on expressed lemmas in [12], the intervehicle spacing is exponentially distributed with the parameter $\lambda_{s}$, then the expected inter-cluster spacing is given by

$$
E\left[S_{\text {inter }}\right]=\frac{1}{\lambda_{s}}+R
$$

Also, if the intervehicle spacing is exponentially distributed with parameter $\lambda_{s}$, the expected number of vehicles in a cluster is

$$
E\left[C_{N}\right]=e^{\lambda_{s R}}
$$

4.3. Average Cluster Length $\left(E\left[C_{L}\right]\right)$. The size of a cluster can also be described by its length between the first vehicle and the last vehicle in a cluster.

If the intervehicle spacing is exponentially distributed with parameter $\lambda_{s}$, then the average cluster length is given as

$$
E\left[C_{L}\right]=\left(\frac{1}{P_{d}}-1\right)\left(\frac{1}{\lambda_{s}}-\frac{R e^{-\lambda_{s} R}}{1-e^{-\lambda_{s} R}}\right) .
$$

The proof of (1), (2), (3), and (6) is amplified in [11].
Let $V_{0}$ be the average speed of every vehicle on the road and $M_{n}(L)$ denote the number of clusters a collector vehicle meets during a travel along a road of length $L$ (note that collector and carrier vehicles move in opposite directions, with respect to an RSU). Further, let $M_{t}$ denote the time duration that a collector vehicle spends in contact with a cluster of carrier vehicles. Given that the intervehicle spacing follows an exponential distribution, $M_{n}(L)$ and $M_{t}$ could be achieved by

$$
\begin{aligned}
& E\left[M_{n}(L)\right] \\
& \quad \approx \frac{2 L}{\left(e^{\lambda_{s} R}-1\right)\left(\left(1 / \lambda_{s}\right)-\left(R e^{-\lambda_{s} R} /\left(1-e^{-\lambda_{s} R}\right)\right)\right)+R+\left(1 / \lambda_{s}\right)},
\end{aligned}
$$

$$
E\left[M_{t}\right]=\frac{\left(e^{\lambda_{s} R}-1\right)\left(\left(1 / \lambda_{s}\right)-\left(R e^{-\lambda_{s} R} /\left(1-e^{-\lambda_{s} R}\right)\right)\right)+2 R}{2 v_{0}}
$$

The proof of (7) and (8) is mentioned in [16] in detail.

\section{Overview of DMRC}

DMRC suggests the application of a new class of packetlevel coding schemes referred as rateless codes for the reliable and efficient data dissemination in VANETs [14, 15]. Several aspects of rateless codes make them suitable for such applications.

In this strategy, each RSU packetizes its message into smaller data packets of the same size. These packets are then encoded into a set of slightly bigger size using rateless coding. Then the RSU broadcasts the set of encoded packets. Vehicles divided in two groups: collectors and carriers. Collector vehicles are approaching to the specific RSU and try to collect its packets. After receiving the required number of packets, they could decode these packets in order to obtain the message of the RSU. By crossing over each RSU, collector vehicles apply rateless coding on the received message. From this point, they act as Carriers and keep packets in their buffer and broadcast them periodically. In order to the better understanding, Figure 1 shows the difference between collectors and carriers.

In this scheme, each carrier node can potentially carry packets from several RSUs simultaneously. Thus, it can act as a carrier and collector for different RSUs at the same time. Every time a collector node listens to a carrier node it receives packets which are innovative (by the rateless encoding property). The number of sufficient packet by which a collector could perform the rateless decoding is limited to $\ell$. By this strategy, it is showed that because of the limited buffer in vehicles, they could not carry out infinite packets.

The parameter DD (Decoding Distance) is the basic performance metric we consider which can provide insight to the throughput. By collecting sufficient packet, each collector could decode the message of each RSU before entering its communication range (at decoding point). The distance 


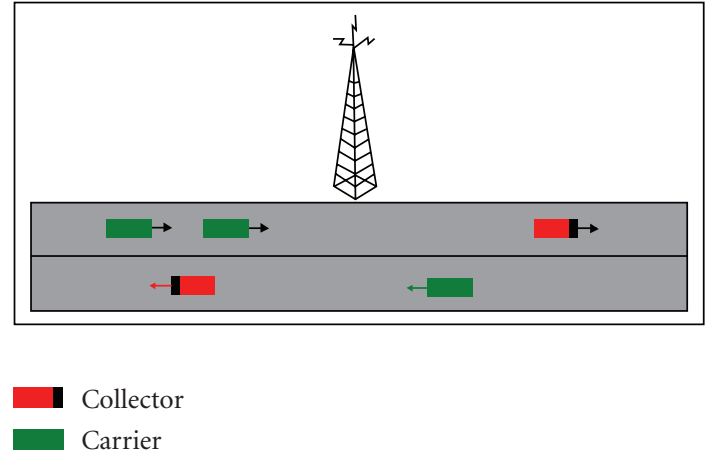

Figure 1: Collector and carrier vehicles.

between each RSU and related decoding point is considered as DD.

To describe DMRC scheme, the basic network model is considered. RSUs are placed uniformly in the road with distance $d$ from each other. The space between two consecutive sources is named as segment. $\phi_{i}$ is representing the $i$ th source in the road. If a vehicle is located in $j$ th segment from source $\phi_{i}$, it is in segment $\phi_{i j}$.

It can be shown that for any source $\phi_{i}, \mathrm{DD}$ is directly proportional to the number of packets from the corresponding source $\left(m_{i, j}\right)$, that carriers posses per each segment $\phi_{i j}$. In order to maximize DD, we need to find a solution for $m_{i, j}$ 's subject to the buffer limit constraint. Since sources are all the same, we can omit the first index in $m_{i, j}$ and find a generic solution $m_{j}$ for all sources. We assume that the number of packets carrier posses from $\phi_{i}$ cannot be increased. Also, buffer updating for a carrier node occurs when it crosses a new source and enters a new segment (e.g., from $\phi_{i j}$ to $\left.\phi_{i j+1}\right)$. Just after crossing $\phi_{i}$ the carrier node has $m_{0}$ encoded packets from the source and reduces them gradually as $\cdots \leq m_{2} \leq m_{1} \leq m_{0}$ and $m_{j}=0$ for $j \geq \Delta$. By considering $N_{j}$ as the total number of collected packets in segment $j, \mathrm{DD}$ can be formally stated as

$$
\begin{gathered}
\mathrm{DD}=\min _{m_{\mathrm{j}}} d, \\
\text { S.t. } \quad \sum_{i=0}^{d} E\left[N_{\Delta-i}\right] \geq \ell .
\end{gathered}
$$

Since [7] suggests that only the tail behavior of the distribution of $m_{j}$ 's is important and because $m_{j}$ is nonincreasing with $j$, one can see that the maximum value of DD is achieved when $m_{\Delta}$ (and hence all the previous segments) has its maximum value [16]. Further, the buffer limit constraint implies that $\sum_{i=0}^{\Delta} m_{i} \leq B$. Therefore, a solution can be formulated as

$$
m_{0}=m_{1}=m_{2}=\cdots=m_{\Delta}=\frac{B}{\Delta+1} .
$$

This solution is only applied for a single road with a fixed velocity. To find the maximum distribution for $m_{j}$ 's in an urban area, we set the desired value for $\mathrm{DD}\left(\mathrm{DD}^{*}\right)$ and calculate the value of $m_{0}$ for all sources.

\section{Proposed Approach for Data Dissemination}

6.1. Proposed Scheme. As mentioned previously, we are interested in solving the problem of distributing large amount of data from multiple sources in an urban area. A network in which roads are separated by junctions is considered. Each road has its own characters. Characters of each road are defined as the average speed of vehicles and the rate of vehicle entering in the road. These two parameters determine the traffic load in each road. RSUs in such a network are uniformly distributed along the roads. Upon crossing an RSU, every node that has been successful in decoding the RSU's message acts as a carrier for that source. Then, every carrier node generates some encoded packets from the RSU's information packets and stores them. The number of stored packets is determined for maximum performance, given that the storage buffer is limited to $B$.

Upon crossing the $i$ th source, every carrier node generates some encoded packets and puts $m_{i, j}$ packets in their buffer in $j$ th segment from source $\phi_{i}$. In the basic model, segments have the same length of $d$. The number of stored packets is determined for maximum performance, given that the storage buffer is limited to $B$. Each collector could gather packets from the vehicle clusters. Consider a collector vehicle meets a cluster of vehicles in $\phi_{i j}$. The number of broadcast packets during the meet time $M_{t}$ follows the Poisson distribution with the mean $\rho$, because carriers send encoded packets randomly and without coordination. Thus, based on [13], the maximum throughput occurs when $\rho$ is equal to $1 / 2$ of packet transfer time and is equal to $1 / 2 e$. By using (6) and (7) and considering that a received packet is of collector's interest (i.e., the packet is from $\phi_{i}$ ) with the probability of $m_{i, j} / B$, the maximum expected number of collected packets from a cluster $\left(N_{j}^{C}\right)$ and total number of collected packets $N_{j}^{T}$, that could be obtained from the segment $\phi_{i j}$, are given by $\psi$

$$
\begin{gathered}
E\left[N_{j}^{c}\right]=\rho_{\max } \times E\left[M_{t}\right] \times \frac{m_{i j}}{B}, \\
E\left[N_{j}^{T}\right]=E\left[N_{j}^{c}\right] \times M_{n}(d) .
\end{gathered}
$$

To find the maximum distribution for $m_{j}$ 's in an urban area, the desired value for $\mathrm{DD}\left(\mathrm{DD}^{*}\right)$ is set and the value of $m_{0}$ for all sources would be calculated.

A network in which the velocity of vehicles is $V_{1}$ is considered. We are interested in achieving the message of RSUs at DD* from each source. The goal is to find the value of $m_{i 0}$ for the $i$ th source. $N$ is considered as the number of RSUs that a carrier node keeps their packets in its buffer. Based on DMRC scheme, $N=B / m_{i 0}$. The value of $m_{i 0}$ for all sources could be obtained as follows:

$$
\rho_{\max } \times E\left[M_{t}\right] \times \frac{m_{i 0}}{B} \times M_{n}\left(N \times d-d^{*}\right)=\ell .
$$

Using (7) and (8) in (12), the value of $m_{i 0}$ for each source would be calculated. Now, in order to find a suitable distribution for an urban area, a large road consisting of segments with different traffic loads is considered. In each segment, the average speed of vehicles and the rate of vehicle 
entering the segment are different. By considering the specific value for $\mathrm{DD}^{*}$ and based on vehicle velocity and intervehicle spacing in each segment of the road, we can calculate the required number of stored packets in the collector buffer corresponding to each RSU. The value of $\mathrm{DD}^{*}$ determines the segment (or segments) in which collector could collect the required number of packets. We assume that the nature of information is commercial advertisement and it is only useful in a nearby geographical area. By this assumption, we could consider the end for our simulated road. Based on the parameters of $j$ th segment, $E\left[N_{j}^{c}\right]$ would be determined. The assumption is that messages from sources located in the last segment are collected by the collectors in that segment. Therefore, the value of $m_{i 0}$ for these sources will be calculated using (12). For these sources, $m_{0 i}=\Delta$ is assumed.

Using the value of $m_{i 0}$ for sources located in the last segment $(\Delta)$, the value of $m_{i 0}$ corresponding to the other sources should be calculated one by one. Therefore, the value of $m_{0}$ for the last source in the last segment but one $\left(m_{0 j}\right)$ is calculated. The number of sources located in the range of $\mathrm{DD}^{*}$ from the above source is defined as $k$. As a result, $\left(\left(B-m_{0 j}-K \Delta\right) / \Delta\right) \times d$ determines the distance in which the collector collects packets for the $j$ th source. Consequently, the value of $m_{0 j}$ is calculated using the following equation:

$$
\rho_{\max } \times E\left[M_{t}\right] \times \frac{m_{0 j}}{B} \times M_{n}\left(\frac{\left(B-m_{0 j}-K \Delta\right)}{\Delta} \times d\right)=\ell .
$$

It should be notified that the value of $E\left[M_{t}\right]$ is varied for each segment. In (13), $E\left[M_{t}\right]$ is calculated based on the parameters of the last segment. Based on the above algorithm, the value of $m_{0}$ for other sources could be calculated, too. The point is that, for each source, the parameters of segments in which the collector collects packets should be determined. Regarding these values, (13) would be altered for each source. For example, for some sources, the last segment may not be included in the distance in which the packets are collected. Accordingly, instead of using $\Delta$ and $k$ for calculating their $m_{0}$, the $m_{0}$ and $k$ values should be determined corresponding to the source of the other segments.

Based on these extracted values for all sources, the trend of dropping packets from the buffer of carriers could not follow the expressed algorithm in DMRC scheme.

The algorithm of the proposed scheme for urban area changes as follows: upon crossing new source in the road $i$, the value of $m_{0 i}$ packets from corresponding source would be stored in the buffer. In order to fix the number of $B$ (buffer size) packets in the buffer, by reaching a new source, collector vehicle should drop a number of old packets which are equal to a number of added packets from the new RSU. Based on this scheme, we find an adaptive solution with the traffic load diversity in an urban area.

6.2. Siimulations. Evaluating the performance of a proposed scheme will be done by developing NS-2 [17] simulator in this section. Implementing the realistic traffic models is performed by SUMO [12]. Vehicles enter the road from
TABLE 1: Simulation parameters.

\begin{tabular}{lc}
\hline Parameters & Value \\
\hline Simulation time & 1600 seconds \\
Communication range $(R)$ & $200 \mathrm{~m}$ \\
Distance between consecutive RSUs $(d)$ & $400 \mathrm{~m}$ \\
Simulation road length & $24000 \mathrm{~m}$ \\
Broadcast interval & $100 /$ second \\
Buffer size & 300 \\
Required number of packet for decoding the & 800 packets \\
message $(\ell)$ &
\end{tabular}

TABLE 2: The values of $m_{0}$.

\begin{tabular}{lc}
\hline Vehicle velocity & $m_{0}$ \\
\hline $20(\mathrm{~m} / \mathrm{s})$ & 11 \\
$30(\mathrm{~m} / \mathrm{s})$ & 8 \\
$40(\mathrm{~m} / \mathrm{s})$ & 4 \\
\hline
\end{tabular}

one end with interarrival times drawn using instances of exponential distribution with parameter $\lambda_{s}$. In order to compare our scheme with DMRC, we also considered 50 points as the positions of RSUs along the road. We use the transmission range $R$ as the unit of distance in our simulations. We focus on a collector vehicle that departs from one end of the road and travels along it until it reaches the other end. The number of vehicles in each road is determined by (6) and (7). Table 1 shows the parameters used in simulations.

As the first step, we investigate the influence of vehicle velocity and interarrival time on determining the number of stored packets in the buffer of carrier corresponding to each RSU $\left(m_{0}\right)$. Primarily, we fix the value of 0.2 vehicle/sec for interarrival time and $2800 \mathrm{~m}$ for $\mathrm{DD}^{*}$. By setting the vehicle velocity and using (12), we can find the values of $m_{0}$ corresponding to each source. These values related to each velocity are presented in Table 2 .

We perform simulations with value determined in Table 2. At this point, we consider another performance metric to compare the result. $P_{\text {success }}$ is the probability that a random message is generated at a (random) source $\phi_{i}$ is available at node $v$ before it enters the communication range of $\phi_{i}$. We measure $P_{\text {success }}$ as a function of distance to the source. The presented graphs are the average of $P_{\text {success }}$ over all active sources.

Figure 2 presents $P_{\text {success }}$ for various vehicle velocities in the road.

Now, in order to see the influence of interarrival time of vehicles, we change it based on the presented values in Table 3 and calculate the value of $m_{0}$ for sources. of $\lambda_{t}$.

Figure 3 shows the changes in $P_{\text {success }}$ for different values

In order to implement our scheme, we consider a 24000 meters road with 2 intersections. The first segment is regarded as the first 6000 meters distance of the road. The second segment is set from 6000 meters till 12000 meters of the road and after 12000 meters is the last segment. Each 


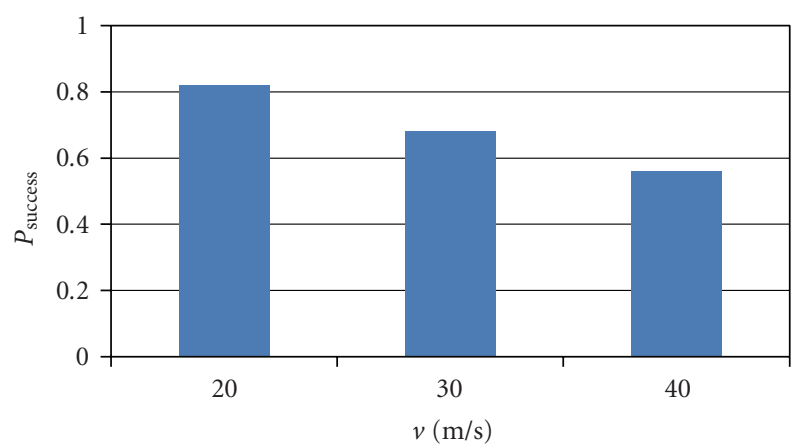

Figure 2: $P_{\text {success }}$ for different velocity.

TABLE 3: The values of $m_{0}$.

\begin{tabular}{lc}
\hline Interarrival time & $m_{0}$ \\
\hline 0.1 (vehicle/sec) & 9 \\
0.3 (vehicle/sec) & 11 \\
$0.4($ vehicle/sec) & 10 \\
0.5 (vehicle/sec) & 9 \\
\hline
\end{tabular}

TABLE 4: Road parameters.

\begin{tabular}{llc}
\hline Segment & Road parameters & Value \\
\hline \multirow{2}{*}{$0-6000 \mathrm{~m}$} & Interarrival of vehicles $\left(\lambda_{1}\right)$ & $\begin{array}{c}0.2 \mathrm{veh} / \mathrm{sec} \\
38 \mathrm{~m} / \mathrm{s}\end{array}$ \\
& Average velocity $\left(V_{1}\right)$ & $0.6 \mathrm{veh} / \mathrm{sec}$ \\
\multirow{2}{*}{$6000-12000 \mathrm{~m}$} & Interarrival of vehicles $\left(\lambda_{2}\right)$ & $28 \mathrm{~m} / \mathrm{s}$ \\
\hline \multirow{2}{*}{$12000-24000 \mathrm{~m}$} & Average velocity $\left(V_{2}\right)$ & $0.4 \mathrm{veh} / \mathrm{sec}$ \\
& Interarrival of vehicles $\left(\lambda_{3}\right)$ & $35 \mathrm{~m} / \mathrm{s}$ \\
\hline
\end{tabular}

TABLE 5: The vaiues of $m_{0}$ for all sources.

\begin{tabular}{ll}
\hline$m_{050}-m_{030}$ & 18 \\
$m_{029}-m_{027}$ & 20 \\
$m_{026}-m_{023}$ & 19 \\
$m_{022}-m_{020}$ & 21 \\
$m_{019}-m_{016}$ & 16 \\
$m_{015}-m_{013}$ & 17 \\
$m_{012}-m_{010}$ & 18 \\
$m_{09}-m_{08}$ & 16 \\
$m_{07}-m_{01}$ & 10 \\
\hline
\end{tabular}

segment is characterized with interarrival of vehicles and the average velocity of vehicles in that segment. Parameters related to each segment are presented in Table 4 .

In order to meet the optimal distribution of $m_{0 i}$, the value of $\mathrm{DD}^{*}$ is set to $2800 \mathrm{~m}$. the value of $m_{0 i}$ corresponding to the sources of the last segment of the road would be calculated by using $N=B / m_{0 i}$ in the (12). Therefore, The value of $m_{0 i}$ for the last 20 sources is equal. Then, we used (13) to adjust the value for 29th source. Considering the value of $\mathrm{DD}^{*}$, we can find the segments in which the collector should collect the packets of specific RSU. The value of $m_{0 i}$ corresponding to each source is presented in Table 5.

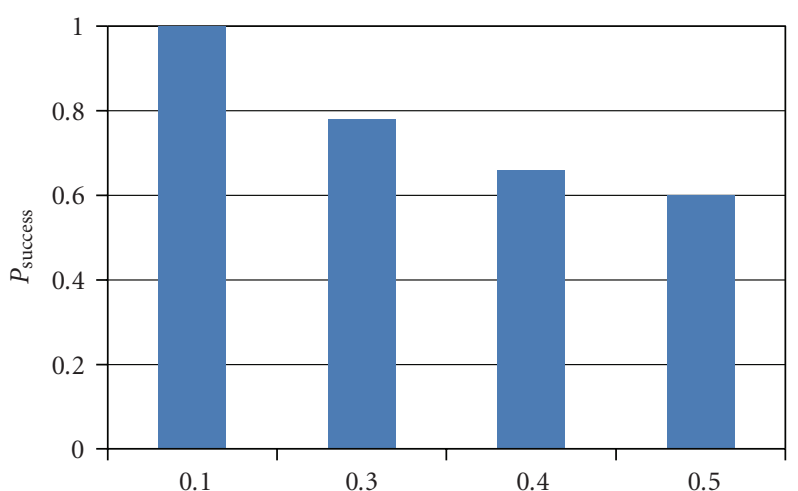

Figure 3: $P_{\text {success }}$ for different $\lambda_{t}$.

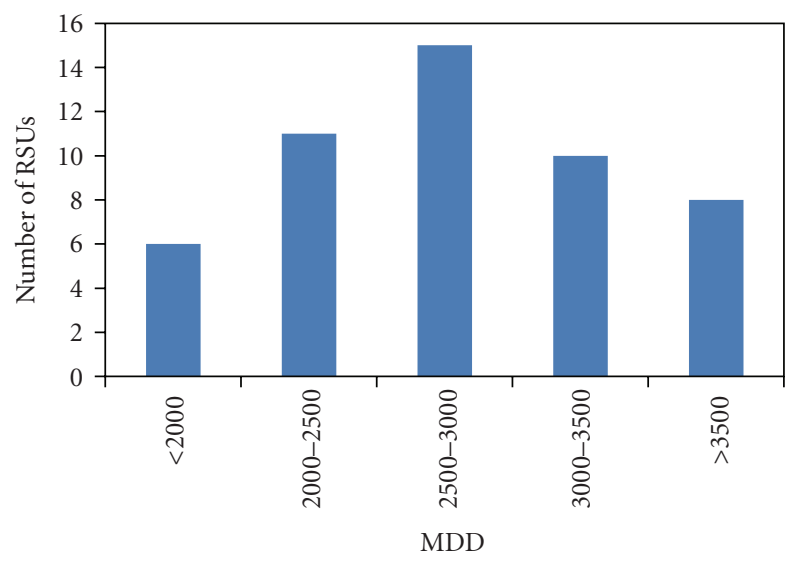

FIGURE 4: Dispersal of MDD around $2800 \mathrm{~m}$ for scheme B.

By considering the value of $N=10$ in DMRC scheme, the value of MDD for vehicle velocity between $20-40(\mathrm{~m} / \mathrm{s})$ is between $3500-2000 \mathrm{~m}$. Therefore, we approximately could mention the average of $2800 \mathrm{~m}$ as decoding distance for these velocities. We compare our scheme (scheme A) with two other schemes. The first is DMRC scheme with $N=10$. The second one is the scheme which uses the average velocity of $V_{i}$ as the velocity and average $\lambda_{i}$ in these three segments as the intervehicle spacing and set these values in (12) to obtain equal value for $m_{0 i}$ corresponding to all sources (scheme B). Based on the determined parameters in Table 2, we have $\bar{v}=33.66 \bar{\lambda}=0.4$. By using (13) and $N=B / m_{0 i}$, we obtain 18 packets as the number of stored packets from every RSU in the road. We first perform the simulation for our proposed scheme.

Figure 4 shows the dispersal of MDD for all sources in the entire road.

Figure 5 shows the dispersal of MDD for all sources in the entire road for the scheme using the average of values in Table 2.

As presented in Figure 4, messages of 15 RSUs are decoded approximately in $2800 \mathrm{~m}$ but in Figure 5, this value is 9. Also the average decoding distance for all RSUs in scheme A is equal to $2814 \mathrm{~m}$ with standard deviation of 770 , but in the scheme $\mathrm{B}, \mathrm{MDD}$ is equal to 3011 with standard deviation of 1181 . Although the larger MDD is the better 


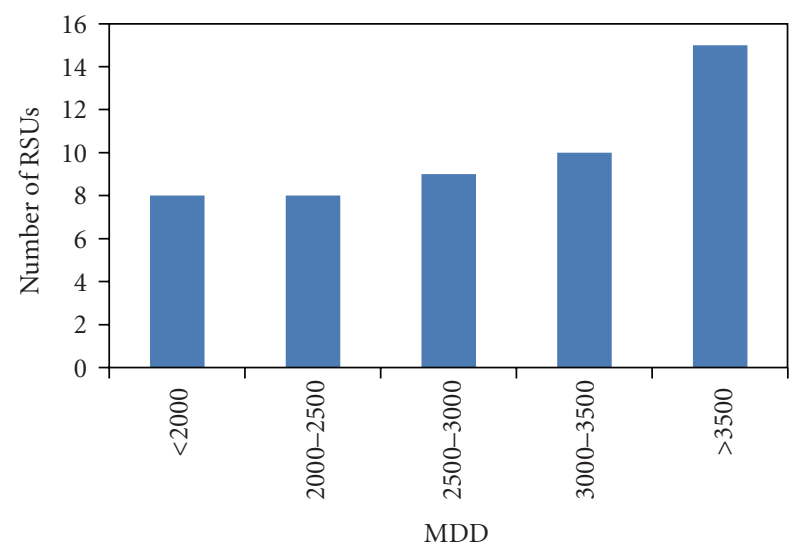

Figure 5: Dispersal of MDD around $2800 \mathrm{~m}$ for scheme B.

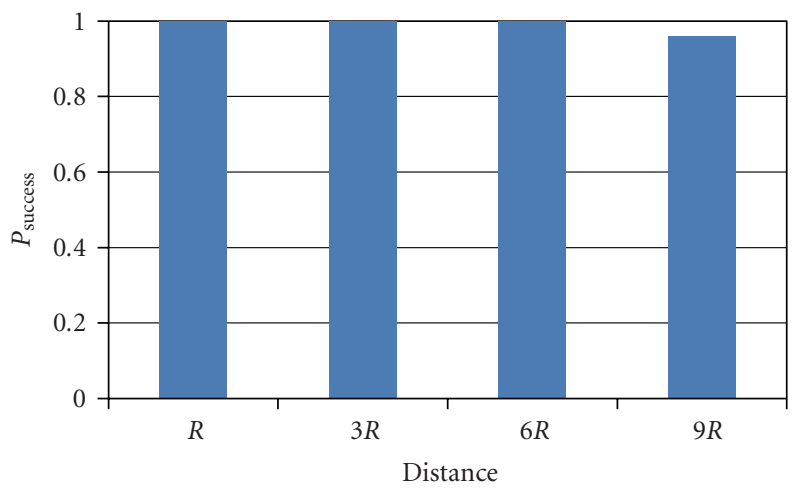

Figure 6: $P_{\text {success }}$ for variouse distances (in multiple of $R$ ) in scheme A.

result, it was expected to find the value of 2800 for MDD. Therefore, our scheme is working better.

In order to evaluate scheme A and DMRC, The value of $P_{\text {success }}$ is compared for both schemes and $P_{\text {success }}$ versus distance is plotted for scheme A and DMRC with $N=10$ in Figures 6 and 7, respectively.

As presented in Figures 6 and 7, $P_{\text {success }}$ in the scheme A has the better results.

\section{Conclusion}

In this work, a new scheme based on rateless codes for collaborative content distribution from road side units to vehicular networks in an urban area is presented. Using the advantage of DMRC, an adaptive model which is compatible with the characteristics of the road was introduced. The proposed scheme can seamlessly handle both sparse and dense scenarios. Our simulations are performed in an urban area without any traffic lights, and by considering some sideways streets, the traffic loads in the road would be changed. The future researches are to introduce more realistic traffic models for urban areas with traffic lights where the exponential assumptions for interarrival time are not valid. Further, adapting the analysis for such scenarios

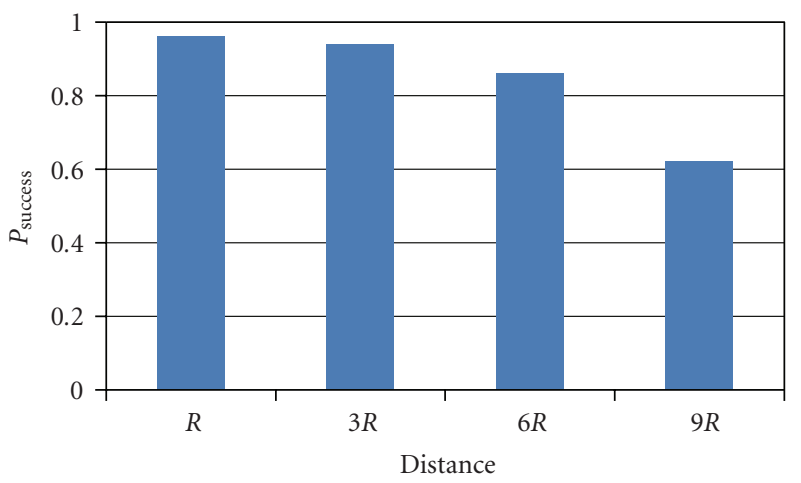

FIgURE 7: $P_{\text {success }}$ for variouse distances (in multiple of $R$ ) in DMRC $(N=10)$.

and finding the optimal distribution for buffer allocation are our immediate goals.

\section{References}

[1] J. A. Davis, A. H. Fagg, and B. N. Levine, "Wearable computers as packet transport mechanisms in highly-partitioned ad-hoc networks," in Proceedings of the 5th International Symposium on Wearable Computers (ISWC 2001), pp. 141-148, October 2001.

[2] L. Wischhof, A. Ebner, and H. Rohling, "Information dissemination in self-organizing intervehicle networks," IEEE Transactions on Intelligent Transportation Systems, vol. 6, no. 1, pp. 90-101, 2005.

[3] J. Zhao and G. Cao, "VADD: vehicle-assisted data delivery in vehicular ad hoc networks," in Proceedings of the 25th IEEE International Conference on Computer Communications (INFOCOM '06), April 2006.

[4] ASTM E2213-03, "Standard specification for telecommunication and information exchange between roadside and vehicle systems $-5 \mathrm{GHz}$ band dedicated short range communications (DSRC) medium access control (MAC) and physical layer (PHY) specificifications," ASTM Int'l, July, 2003.

[5] J. Yin, T. Elbatt, G. Yeung et al., "Performance evaluation of safety applications over DSRC vehicular ad hoc networks," in Proceedings of the 1st ACM International Workshop on Vehicular Ad Hoc Networks (VANET '04), pp. 1-9, October 2004.

[6] S. B. Lee, G. Pan, J. S. Park, M. Gerla, and S. Lu, "Secure incentives for commercial ad dissemination in vehicular networks," in Proceedings of the 8th ACM International Symposium on Mobile Ad Hoc Networking and Computing (MobiHoc '07), pp. 150-159, New York, NY, USA, September 2007.

[7] M. Sardari, F. Hendessi, and F. Fekri, "DMRC: dissemination of multimedia in vehicular networks using rateless codes," in Proceedings of the IEEE International Conference on Computer Communications (INFOCOM '09), pp. 1-6, April 2009.

[8] V. Namboodiri, M. Agarwal, and L. Gao, "A study on the feasibility of mobile gateways for vehicular ad-hoc networks," in Proceedings of the 1st ACM International Workshop on Vehicular Ad Hoc Networks (VANET '04), pp. 66-75, October 2004.

[9] H. Wu, R. Fujimoto, R. Guensler, and M. Hunter, "MDDV: a mobility-centric data dissemination algorithm for vehicular 
networks," in Proceedings of the 1st ACM International Workshop on Vehicular Ad Hoc Networks (VANET '04), pp. 47-56, October 2004.

[10] Z. D. Chen, H. T. Kung, and D. Vlah, "Ad hoc relay wireless networks over moving vehicles on highways," in Proceedings of the ACM International Symposium on Mobile Ad Hoc Networking and Computing (MobiHoc '01), pp. 247-250, October 2001.

[11] N. Wisitpongphan, F. Bai, P. Mudalige, V. Sadekar, and O. Tonguz, "Routing in sparse vehicular ad hoc wireless networks," IEEE Journal on Selected Areas in Communications, vol. 25, no. 8, pp. 1538-1556, 2007.

[12] "Simulation of urban mobility," http://sumo.sourceforge.net/.

[13] N. Abramson, "The Aloha System: another alternative for computer communications," in Proceedings of the Fall Joint Computer Conference (AFIPS '70), pp. 281-285, Montvale, NJ, USA, 1970.

[14] M. Luby, "LT codes," in Proceedings of the 34rd Annual IEEE Symposium on Foundations of Computer Science, pp. 271-280, 2002.

[15] Y. Wang, S. Jain, M. Martonosi, and K. Fall, "Erasure-coding based routing for apportunistic networks," in Proceeding of the ACM SIGCOMM Workshop on Delay-Tolerant Networking (WDTN '05), pp. 229-236, New York, NY, USA, 2005.

[16] M. Sardari, F. Hendessi, and F. Fekri, "Infocast: a new paradigm for collaborative content distribution from roadside units to vehicular networks," in Proceedings of the 6th Annual IEEE Communications Society Conference on Sensor, Mesh and Ad Hoc Communications and Networks (SECON '09), June 2009.

[17] “The Network Simulator NS-2," http://www.isi.edu/nsnam/ ns/. 

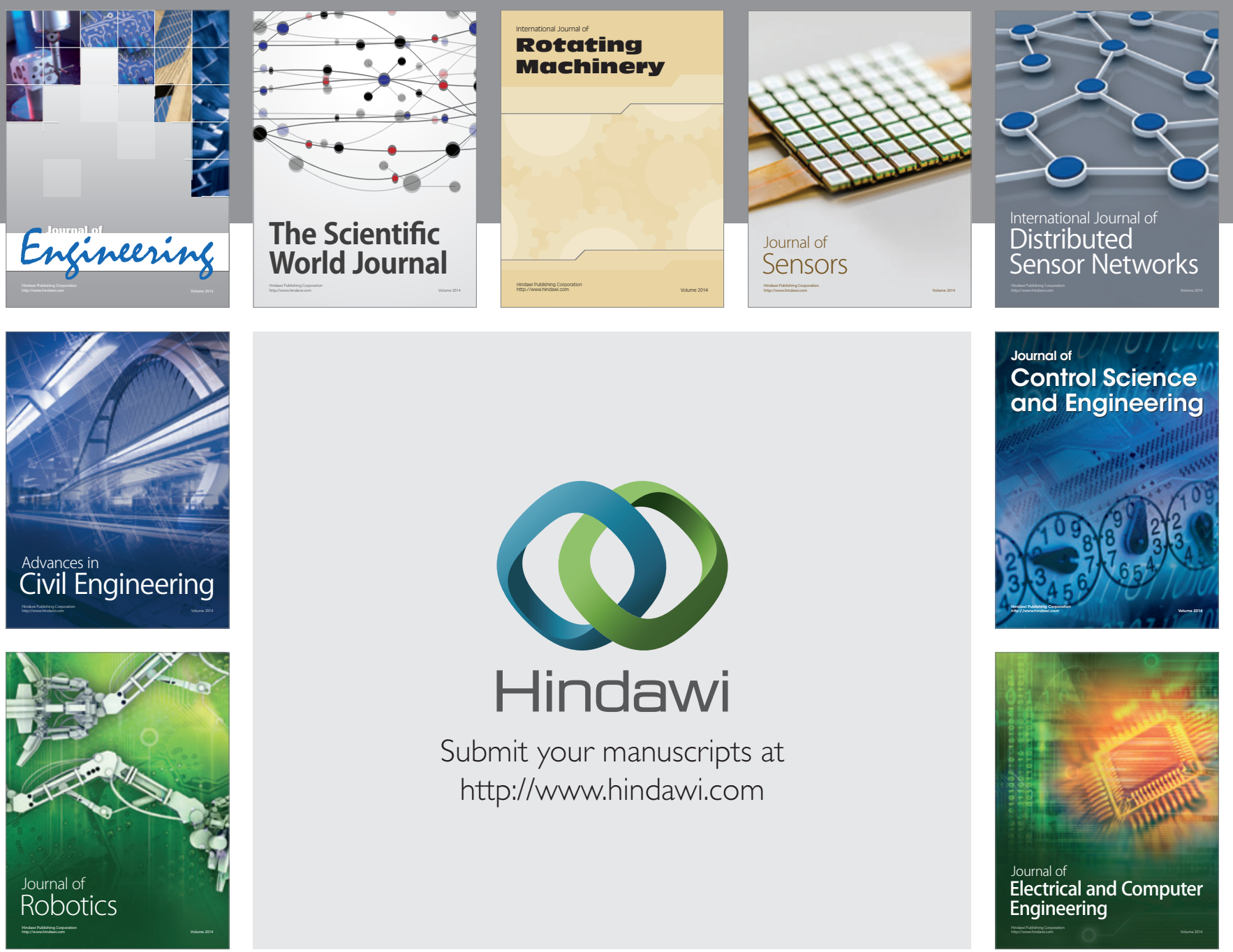

Submit your manuscripts at

http://www.hindawi.com
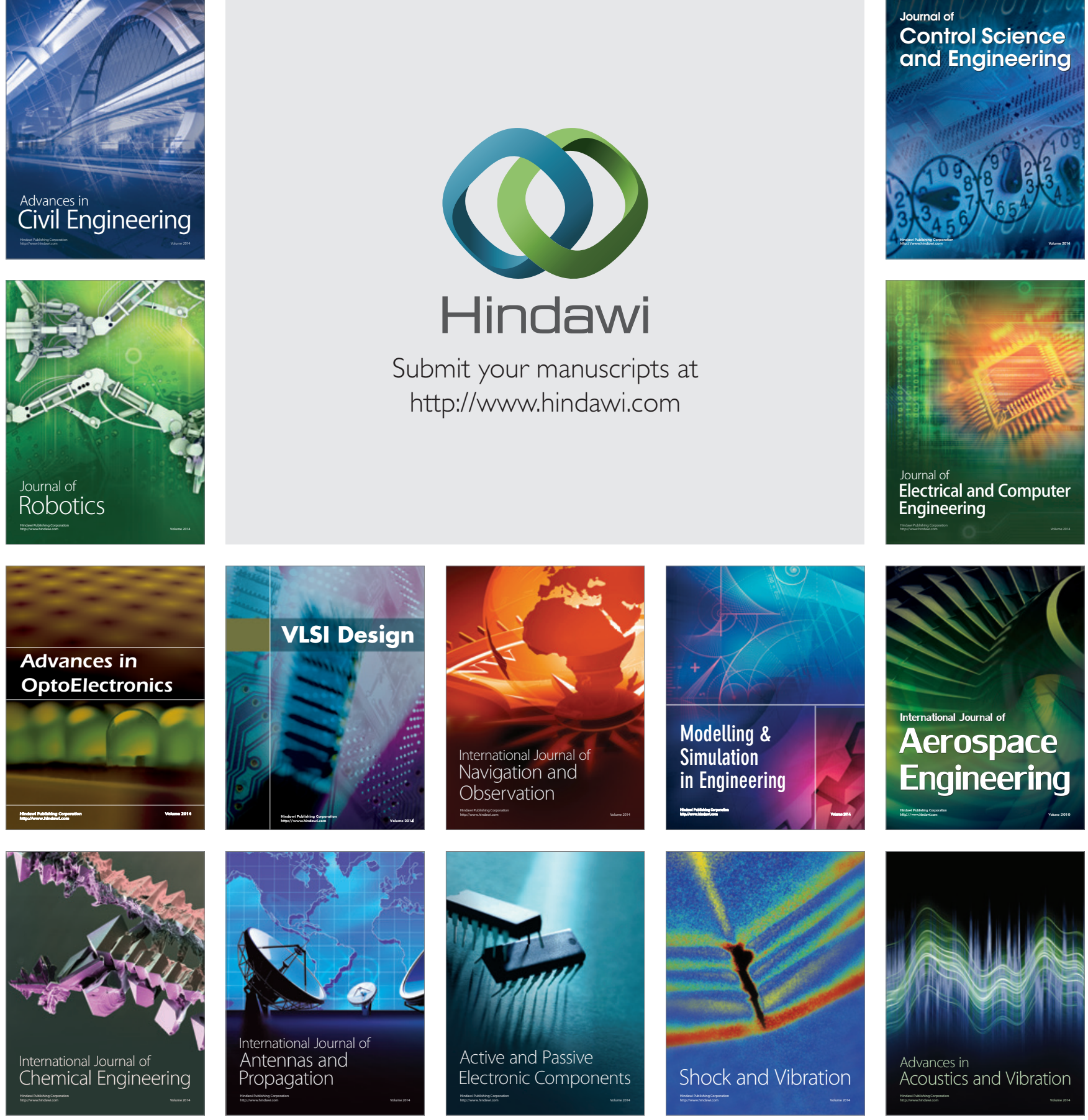